\section{LAS REDES SOCIALES DIGITALES COMO MARCO DE UN NUEVO PARADIGMA EN EL ARTE CONTEMPORÁNEO}

\author{
Violeta Izquierdo Expósito \\ Universidad Complutense de Madrid \\ violeta.izquierdo@ccinf.ucm.es
}

SOCIAL DIGITAL NETWORIS AS A FRAME FOR A CONTEMPORARY ART'S NEW PARADIGM

Para citar este artículo:

Izquierdo Expósito, V. \& Lima Guerrero, D. (2018). Las redes sociales digitales como marco de un nuevo paradigma en el arte contemporáneo. Commons. Revista de Comunicación y Ciudadanía Digital, 7(2), 67-94

http://dx.doi.org/10.25267/COMMONS.2018.v7.i2.03

Fecha de recepción: 17/09/2018. Fecha de aceptación: 2/11/2018

\section{Resumen}

La combinación del binomio existente entre arte y redes sociales digitales ha dado lugar a una potente transformación en el panorama de la creación artística desde la década de 1990. Es por tanto preciso concretar y delimitar las relaciones y los efectos que ha provocado esta asociación. Las dos premisas que rigen el objeto de nuestra investigación se reparten en torno a la exploración de cómo las redes sociales digitales se convierten en material para la creatividad, llegando en ocasiones a trasgredir los límites de Internet y materializarse y, por otro lado, el análisis de cómo estas redes llegan a edificarse como oportunidad para la difusión de las obras, contribuyendo a la construcción de un inédito paradigma creativo en el marco del denominado new media art.

\author{
David Lima Guerrero \\ Universidad Complutense de Madrid \\ dalima@ucm.es
}

\section{Palabras clave}

Arte, digital, comunicación, redes sociales, new media art

\begin{abstract}
The combination of the existing binomial between art and digital social networks has led to a powerful transformation in the artistic creation landscape since the 1990s. Therefore, it is necessary to specify and delimit relationships and effects which this association has caused. The two leading premises of our research are divided around the exploration of how digital social networks become material for creativity, sometimes reaching beyond the limits of the Internet and materialize and, on the other hand, the analysis of how these networks come to be built as an opportunity for the dissemination of works, contributing to the construction of an unprecedented creative paradigm in the framework of the so-called new media art.
\end{abstract}

\section{Keywords}

Art, digital, communication, social networks, new media art 


\section{Introducción}

Los contextos y las manifestaciones relacionadas con el arte contemporáneo han experimentado una profunda transformación en las últimas décadas. Muchos de ellos han germinado a una velocidad paralela a la de las nuevas tecnologías del marco digital. Sobre estas líneas planteamos analizar y estudiar la relación existente entre un espacio específico de estas últimas - las denominadas redes socialesy el incipiente desarrollo de formas artísticas desde la década de 1990 hasta la actualidad, en el marco de la sociedad global. El objetivo, por tanto, será definir la relación entre ambos polos, para observar cómo dan lugar a la configuración del concepto de "prácticas artísticas en redes sociales digitales", como fenómeno concreto ligado al siglo presente.

Para comprender la lógica relacional entre las redes sociales y el arte de las últimas décadas deberíamos alcanzar una comprensión de la lógica que ha ido induciendo a la colaboración de ambos elementos, hasta integrarse en un todo que hace posible la creación artística. ¿Cuáles son, por tanto, los precedentes de tal relación antes del desbordamiento del desarrollo tecnológico a nivel estructural? Parece que la relación ya ha sido señalada por diversos autores. Azam y Federico (2014: 3) ponen de manifiesto cómo, en el análisis realizado por Pierre Bourdieu sobre la figura de Flaubert en Las reglas del arte (1995), los actores implicados en un campo de creación artística se relacionan de tal manera que dicha relación influye en la construcción de las obras y en las consecuencias que de estas derivan. Por otro lado, los mismos autores subrayan que este concepto de red se asocia también a "la base del concepto de capital social que hace referencia al conjunto de personas que un individuo puede movilizar para tener acceso a un recurso" (Ibíd.). De esta manera, podemos establecer un nexo entre la importancia de las características que se han dado tradicionalmente en las relaciones sociales de los círculos de creación - ahora como elemento decisivo y condicionante- y el desarrollo de las creaciones en su proceso de gestación. 
El marco de las redes sociales como fuente de conexión entre individuos ha alcanzado un alto reconocimiento. A raíz de ello, ha surgido una "sociología de las redes sociales (...) como una especialidad particular" (Ibíd., 2014: 5). Las aproximaciones recientes revelan la particularidad de este ámbito de estudio como origen para entender nuevos procesos de interacción social desarrollados en el ámbito contemporáneo:

\section{(...) las redes ofrecen la posibilidad de pensar los fenómenos sociales de otra forma, más allá de la articulación entre el nivel macro sociológico de las instituciones y el nivel micro sociológico de los individuos, proponiendo una aproximación frecuentemente calificada de meso-social. (Ibíd., 2014: 6)}

Sin embargo, hasta cerca de la década del 2000 no se pudo llegar a una conciliación entre sociología del arte y sociología de las redes sociales. Como afirmaron Azam y Federico (2014), es necesaria una conciencia sobre el problema generacional que se plantea a la hora de abordar los estudios. La aproximación a esta parcela de trabajo requiere, ante lo mencionado, un conocimiento que parta de la experiencia propia en el medio, de una sensibilidad específica; será preciso que el propio investigador tome parte en el nuevo paradigma, manejando la comprensión de un medio en el que se integran fenómenos artísticos en formas muy diversas.

Una vez situados en el contexto de las redes sociales digitales, encontramos cómo serán un punto germinal de transformación sobre la figura del profesional del arte, además de determinar la naturaleza de su obra. La relación de dependencia entre este entorno tecnológico y el desarrollo del arte en este momento histórico ha sido ya puesta de relieve por investigadores de todos los campos. El antropólogo Jacinto Choza (2015) ha desarrollado una explicación al problema contemporáneo que denomina "Teoría del Interfaz", una visión del contexto actual en el que cobra especial importancia la primacía de las pantallas como medio de vertebración de las comunicaciones en las sociedades actuales. Para Choza, el arte del tiempo presente se ramificará en torno a tres ejes: diseño, publicidad y moda. Más allá de la especifidad con la que el autor recoge su enfoque sobre el arte actual, nos interesa 
reparar en la forma en que concibe la relación entre arte y comunicación en nuestros días. De forma más acentuada que nunca, muchos de los nuevos contextos artísticos encuentran su origen y desarrollo en las propiedades comunicativas que se derivan del uso de las tecnologías que usan Internet, hasta llegar al ámbito específico en el que nos pretendemos mover aquí: las redes sociales en línea. De este modo, plantea Choza una aproximación conceptual al fenómeno del siglo XXI respecto a esta cuestión:

\begin{abstract}
El interfaz es una pantalla. Es la parte visible de un utensilio informático a través del cual se recibe información de cualquier escenario o mundo, mediante el cual se intercambia información con cualquier escenario o mundo. Desde comienzos del siglo XXI la comunicación transcurre fundamentalmente a través de una pantalla y en interacción con ella, a través de tres tipos de pantalla: la pantalla de televisión, la pantalla del ordenador y la pantalla del teléfono móvil. (...). El interfaz aparece así como el utensilio mediante el cual el universo y la sociedad entera se convierten en materia de la interacción artística comunicativa. (Choza, 2015: 366)
\end{abstract}

El arte ha cambiado de lugar, ha flexibilizado sus fronteras, y se ha diluido en una serie de parámetros que lo están transformando, tales como la policontextualidad y la multimedialidad. No creemos adecuado hablar de una "muerte del arte" como se ha venido anunciando en numerosas ocasiones. Se trata de una metamorfosis, una aceleración de los tiempos.

Si bien es cierto que el eje de lo tecnológico es crucial en este nuevo paradigma de infraestructura comunicacional, debemos mirar con detenimiento al condicionamiento que realiza la macroestructura económica, que se presenta como otro eje condicionante de la actividad artística. Los creadores se encuentran en un mercado marcado por una elevada competencia, aunque con más herramientas a su disposición que nunca. Jiménez (2010) realiza una interesante valoración de la remodelación del profesional del arte en el marco actual: 
El arte no muere, no desaparece. Pero queda digerido en ese inmenso aparato digestivo de la cultura de masas. Como un efecto de esa degradación está la existencia y ampliación de un universo de $<<$ mediadores $>>$, de $<<$ profesionales del arte $>>$. Lo que podría constituir un importante elemento en la extensión social, educativa, del arte, deriva en muchas ocasiones, en cambio, hacia el glamour y la agitación propagandística. (...) En ese universo, el papel de los artistas se ve reformulado de un modo radical. En lugar de la leyenda del bohemio, del rebelde inadaptado, el artista se convierte en una especie de agente cultural, relaciones públicas y técnico de la comunicación, todo a la vez, para poder conseguir el acceso al circuito institucional. (2010: 239)

Junto a esta comprensión de la circunstancia tecnológica que afecta al ámbito de lo comunicacional y, por tanto, al desarrollo del arte, podemos abordar la variación de lo estético a través de enfoques igualmente recientes. Nos referimos a la variedad de propuestas que, como herederas del siglo XX, parecen asomarse en los albores del nuevo siglo (Marchán Fiz, 2012: 247). La utilización de redes sociales dará lugar a un desarrollo de propiedades estéticas propias asociadas a la forma en que los usuarios interactúan con ellas, y el nuevo creador lo contemplará en el desarrollo de su obra. Así, a partir de las indagaciones de Claramonte (2016) en su Estética Modal, podemos acercarnos bajo un enfoque diferente a fenómenos artísticos que beben de muy diversas fuentes en la actualidad.

Retomando la problemática surgida de la transición generacional antes enunciada, queremos poner el énfasis en una de las aproximaciones más completas que se han hecho desde los años 90 en los estudios sobre creación cultural y nuevas generaciones en la era de lo digital. En este caso, nos referimos a Jóvenes, culturas urbanas y redes digitales, una publicación coordinada por García, Cruces y Urteaga (2012) en la que se recogen diversos artículos que nos aproximan a un estudio actualizado de las "prácticas emergentes en las artes, las editoriales y la música", partiendo de dos localizaciones concretas: México y España. En sus análisis se puede observar cómo los parámetros a los que nos hemos referido no solo han transformado la escena creativa, sino que han engendrado nuevas formas de vida y de profesionalización completamente diferentes a las de tiempos pretéritos. 
Para García Canclini (2012: 19), —-haciendo énfasis en la transición generacional en la que nos ubicamos-, "las formas industriales y postindustriales de producir y circular los bienes y mensajes conviven con hábitos comunitarios antiguos". En el caso de las redes sociales, estamos hablando de un motor de transformación de la profesión misma del artista. Según el autor, estas nuevas dinámicas condicionadas por las redes darán lugar a la producción de proyectos "inestables y discontinuos".

\section{Marco teórico}

La comprensión de las relaciones sobre prácticas artísticas digitales y comunicación que establecemos en este trabajo emana, en gran parte, de los precedentes estudios sobre la postmodernidad y los nuevos desarrollos de lo estético. En los trabajos desarrollados a partir de los años 50 se puede observar una conclusión generalizada al respecto: la postmodernidad parece poseer, entre otros, un rasgo especialmente identificativo, el proceso de estetización de las distintas parcelas de cotidianidad en la cada vez mayor sociedad globalizada. Frente a medios de comunicación masivos como la televisión, el mundo digital ofrece una relación nunca experimentada entre usuario y contenido. El "tiempo real" aparece suprimido y sustituido por un "tiempo virtual", de modo que la experiencia vivencial del individuo continúa al otro lado de la pantalla (Baudrillard, 2000: 204-205).

Dado el cambio de relación entre los individuos y el mundo originado por las transformaciones digitales, se hace necesario valorar el arte más actual desde otras perspectivas, que alcancen la conciencia de que no nos encontramos ante una supresión del fenómeno artístico, sino de un cambio en la forma, contenido y desarrollos internos del mismo. En este sentido, conviene señalar la visión que Lipovetsky y Serroy (2016) han aportado a la hora de relacionar el nuevo panorama del arte en el contexto económico capitalista. Así, afirman: "vivimos 
en la época hipermoderna de la mezcla de los géneros, de las transversalidades creativas, de las desregulaciones productivas de relaciones o de síntesis estéticocomerciales" (2016: 64). Nos referiremos, por tanto, a lo largo de esta aproximación, al arte profesionalizado. Con todo, la situación que parece esbozarse hace difícil una delimitación de cuáles son las fronteras que separan al tradicional amateur del artista profesional. El número de individuos dedicados a la industria de la creación artística ha llegado a niveles impensables en tiempos pasados (Ibíd., 2016: 90), y ello parece esbozar el preludio de un conflicto aparentemente en sus inicios. A este respecto, nuestra aproximación nos conduce a encontrar en el nuevo orden creativo un flujo de posibilidad para la creación, de supervivencia en el ecosistema desarrollado, alejándonos, por el momento, de entrar en la dinámica de las distinciones mencionadas.

Para acercarse a la comprensión del nuevo horizonte creativo debemos entender que existe un nexo vinculante entre los elementos que integran los nuevos escenarios de creación, y que conectan las posibilidades de creación del artista con el contexto económico y tecnológico al que pertenecen. Así, podemos incluir en el conjunto la necesidad de subsistencia económica, la aplicación de la creatividad en el marco interno de las redes sociales - con sus herramientas y posibilidades - y el desarrollo de nuevos modos de trabajo y sociabilidad. Todo ello, siguiendo el camino de la necesidad, desemboca en un aumento de la demanda de conectividad, con el fin de aumentar las probabilidades de concluir satisfactoriamente los proyectos propuestos (Urteaga, 2012: 20). A partir de su estudio en referencia a la juventud mexicana, Urteaga (2012: 34) reitera que "los entornos de producción cultural están siendo reconfigurados por varios procesos simultáneos", concepto que ya hemos subrayado.

Gerber y Pinochet (2012) no dudan por su parte en subrayar la naturaleza de los nuevos actores del panorama creativo, definiéndolos y describiéndolos como nuevos productores culturales: 
Ellos desarrollaron un modo no lineal de pensamiento que refleja exactamente el lenguaje de Internet, donde un sinfín de asuntos pueden ser acompañados al mismo tiempo. Para estos millenialls [sic], es natural empezar una cosa y terminar en otra. (...) Hoy día, es cool saber y ser muchas cosas a la vez. Es posible ser surfero, dj, rockero, nerd, cinéfilo y diseñador al mismo tiempo. (2012: 57)

Aunque la reflexión de determinados autores (Baudrillard, 2009) aporta una visión marcadamente crítica ante las posibilidades de desarrollo personal y psicológico de los individuos en el nuevo contexto posmoderno, los enfoques que abogan por un optimismo ante las posibilidades técnicas (Kellner, 2011) dejan abierta la posibilidad de replantear el uso que se puede hacer del mundo digital para el desarrollo del arte.

Llegamos así a esclarecer que para nuestro análisis debemos abordar la transformación que suponen los nuevos medios de interacción comunicativa -en nuestro caso, las redes sociales digitales-, en el perfil de los nuevos creadores, además de la importancia que posee el entorno específico en el que se desenvuelven. Ortega (2012) afirma al respecto:

(...) el ciberespacio existe de manera inmaterial y (...) configura, condiciona e impacta la vida cotidiana de los jóvenes: este, señala Sassen, está regulado a partir de valores, las culturas, los sistemas de poder y las organizaciones institucionales dentro de las cuales se inscriben (Sassen, 2002: 348). De ahí la importancia de responder a la pregunta: ¿en qué sentido el ciberespacio prolonga y complementa el mundo físico? Rheingold (2003) observa que en la actualidad los jóvenes preferentemente habitan el ciberespacio como ámbito privado, permitiéndoles agregarse socialmente como comunidades virtuales. (2012: 125) 
Identificados los ejes de interacción contextual en los nuevos procesos de creación artística, podemos valorar algunos ejemplos emblemáticos de propuestas concretas de creación en relación a las redes sociales digitales. ¿Cuáles serán las tensiones desarrolladas entre ambos elementos? Por un lado, la inclusión de redes sociales digitales delimitará la circunstancia del autor con respecto a sus receptores - su arte se difundirá a través de un medio que constituye una ramificación de su círculo social, con enormes posibilidades que multiplican las variables para la difusión-y, por otro, ejercerán modificaciones directamente en el contenido de las obras - tanto por el medio digital, que posee unas cualidades determinadas, como por la necesidad de convertir la obra en un objeto artístico adaptado a las formas de recepción a través de las pantallas-. La enorme vinculación de la presencia de los desarrollos comunicacionales en convivencia con las estructuras socioeconómicas queda evidenciada de este modo.

Las investigaciones en torno al desarrollo de nuevas formas de arte en consonancia con la utilización de redes sociales han planteado varias dificultades en el seno del ámbito sociológico, casi siempre relacionadas con la metodología empleada. En algunos casos, como se señalaba en Azam y Federico (2014), no se manejaban con soltura los lenguajes empleados en estas áreas tan policontextuales. La brecha generacional complicaba las posibilidades de entendimiento ante el nuevo paradigma. Lipovetsky y Serroy (2009: 306) han señalado cómo la explosión digital nacida a raíz de los años noventa ha dado lugar a un elenco de marcos experimentales sin precedentes. Sin embargo, al contrario que en el contexto cinematográfico —-donde la técnica aparece al servicio del relato emocional一, en esta exploración de formatos artísticos no parece haberse encontrado un equilibrio entre relato y posibilidades técnicas sino, quizá, una exacerbación de la experiencia estética de estas últimas. Coincidimos con estos autores en que nos encontramos en un momento decisivo para el desarrollo de los ecosistemas artísticos de las décadas próximas. 


\section{Metodología}

Dado el objeto de análisis del presente trabajo, el proceso de investigación poseerá un carácter claramente cualitativo, de acuerdo a como Creswell (1994: 1) ha descrito en los supuestos del paradigma cualitativo:

\footnotetext{
(...) definido como un proceso de indagación para la comprensión de problemas sociales o humanos, basado en la construcción de cuadros/descripciones (pictures) formados en base a palabras, dando cuenta detalladamente de la perspectiva de los informantes, y conducido en un escenario natural.
}

El orden estructural de la investigación ha seguido un planteamiento inductivo, partiendo del análisis de ejemplos concretos en la creación artística a partir de los años 90 en el marco de las redes sociales digitales. Más que una identificación de los elementos que configuran el desarrollo concreto de cada obra, se ha tratado de buscar los ejes condicionantes para su desarrollo, de forma que, ya ordenados, permitan una orientación basada en la perspectiva múltiple del fenómeno.

El desarrollo todavía preliminar de este objeto de investigación nos obliga a resaltar la dificultad a la hora de manejar una terminología consensuada entre las diferentes fuentes manejadas. Para Martín Prada (2015), se ha hecho un uso impreciso del término net art, que engloba "aquellas obras para las que las tecnologías basadas en redes de telecomunicación (no solo Internet) son condición suficiente y necesaria para su existencia (...)" (2015: 14). Queda patente la necesidad de establecer una conceptualización precisa en un campo semántico que engloba arte, red social, Internet, telecomunicaciones, web y conexión a la red. Por su parte, Martín Prada da un paso al querer acotar conceptualmente algunas de estas parcelas. 
(...) con el término <<arte de Internet >> señalaremos todas aquellas manifestaciones de net art para las que el acceso a Internet resulta imprescindible para la experimentación o participación en la obra. (...) con el término web art nos referiremos (...) a todas aquellas obras que, dentro del marco general del arte de Internet, operan con el sistema de documentos de hipertexto y/o hipermedios enlazados y accesibles a través de Internet que constituye la World Wide Web, siendo estas tecnologías condición necesaria para la existencia de la obra, así como el acceso a la web imprescindible para poder experimentarla. (Prada, 2015:14-15)

Otro de los elementos que permiten realizar una aproximación adecuada a la cuestión en el presente trabajo es la revisión bibliográfica escogida. Las aproximaciones realizadas desde la década de los 90 han surgido como fruto de la interconexión de investigaciones que integran los estudios sobre comunicación y arte contemporáneo. Sin embargo, nos encontramos ante un campo de especial dificultad a la hora de recavar resultados vinculantes. Esto se debe a la celeridad que experimentan las tecnologías de la red y que obligan a permanecer siempre bajo un análisis policontextual y llevado a cabo desde múltiples perspectivas de estudio. Es en la misma dinámica de acercamiento al fenómeno que realiza la denominada "estética modal" como queremos afrontar nuestra construcción de conclusiones, con el objetivo de mantenerlas adaptadas a la naturaleza del complejo sistema de creación que parece evidenciarse (Claramonte, 2016: 296).

La labor que nos planteamos es explorar a través de casos concretos y comparaciones de análisis los dos ejes principales a los que nos hemos referido: las transformaciones de la circunstancia del autor como creador y los cambios que adoptan los procesos de creación en las obras que precisan de las redes sociales digitales como material de trabajo. En esta dinámica, y de acuerdo con la revisión bibliográfica planteada, se ha incidido en la búsqueda de ejemplos representativos a la hora de ilustrar los casos más significativos. Como ya hemos defendido, continuamos afirmando que la investigación de este objeto de estudio no puede prescindir de una implicación directa en el medio específico en el que se desarrolla, esto es, en las redes sociales digitales. 


\section{Resultados y discusión}

La relación entre arte e Internet se remonta a mediados de los años noventa del siglo XX, momento en el que los artistas intuyeron las posibilidades que ese entorno tecnológico podría aportar a sus investigaciones, además de atisbar que tal medio podría ser un espacio para la creación y la reflexión. Las primeras manifestaciones de arte en Internet conocidas como Net.art fueron proyectos colaborativos vinculados a procesos comunicativos y no tanto enfocados a la realización de obras concretas. La abundante terminología derivada de esta corriente creativa ha supuesto una dificultad añadida para su clasificación y estudio; no obstante, en las recientes investigaciones sobre estas prácticas artísticas ya existe el acuerdo en considerar la necesidad de acceso a Internet como requisito imprescindible para creación de la obra. Manifestaciones como las desarrolladas por un grupo de jóvenes en 1995 bajo el nombre de Net.art group fueron pioneras en la utilización de Internet como espacio de reflexión e intervención.

Estudios recientes sobre la cuestión abren el camino hacia la segunda época del arte en Internet. Así, en torno a 2003, el devenir "social" de la web tras la crisis de las "punto com" y la instauración del nuevo modelo basado en las redes sociales, los principios de participación colectiva abierta, opinión y comentario, es cuando se puede hablar propiamente de una segunda "fase", "periodo" o "época" del arte de Internet (Martín Prada, 2012: 22). De alguna manera estaríamos estableciendo una evolución de aquellas primeras experimentaciones artísticas basadas en las prácticas inmateriales de Internet a un nuevo sistema-red que incluye nuevas dinámicas sociales, generando un contexto diferente y potenciando nuevos usos artísticos derivados de la aparición de redes impulsadas por las tecnologías. 
Podemos afirmar que las redes sociales han pasado a ser, en pocos años, un fenómeno global, expandiéndose como sistemas abiertos y en constante construcción. Su inicial pretensión de conectar personas o entidades unidas por intereses comunes, la participación colectiva o la interacción personal se están enriqueciendo con nuevos usos de las mismas en los distintos ámbitos profesionales. Su importancia es tal que generan nuevas formas de expresión o incluso perfiles laborales especializados en esta tarea. Los ámbitos de la comunicación y la divulgación se han visto sensiblemente afectados por su protagonismo en el contexto socio-cultural; además, estos recursos han modificado la forma de comunicarnos y compartir información. Derivado de todo ello, nos encontramos con un acontecimiento de suma contemporaneidad: han suscitado gran interés en la sociedad, aceptando su uso de forma masiva e incorporándolas en sus costumbres cotidianas (Izquierdo, 2017: 1162).

Las redes sociales digitales (en forma de listas de distribución de mensajes o comunidades virtuales como Geocities y Tripod) han estado presentes desde los primeros años de la World Wide Web, aunque no es hasta principios de la década del 2000 cuando empezaron a adquirir una mayor relevancia y popularidad, con marcos web como Friendster (iniciado en 2002), MySpace y LinkedIn (2003), pero sobre todo a partir de 2004, con la creación de Facebook, YouTube (2005) y Twitter (2006). En enero de 2018, la red social de la compañía Facebook cuenta con 2167 millones de usuarios (según datos de IWS), seguida de YouTube, con 1500 millones, y Twitter, con 330 millones - y una actividad diaria de cientos de millones de posts y tweets diarios-. Estas cifras son sólo una muestra de un hecho evidente: las redes sociales dominan la mayor parte de la actividad en Internet y ejercen una considerable influencia en la vida diaria de la sociedad en los países más desarrollados. 
En este contexto de participación colectiva y utilización de las redes sociales, el arte y los artistas también han encontrado un ámbito atractivo para explorar las posibilidades artísticas que ofrecen tanto a nivel creativo como de difusión de la obra, incluso como canal de reivindicación social, promoviendo prácticas artísticas comprometidas orientadas a plantear cuestionamientos sociales en el sistema-red. Debemos recalcar, antes de iniciarnos específicamente en el análisis del binomio artista/redes sociales, las dos premisas que rigen el objeto de nuestra investigación: por un lado, explorar cómo las redes sociales se convierten en nuevo material estimulador de creatividad, llegando en ocasiones a trasgredir los límites de Internet, hasta llegar a materializarse en fases posteriores en arte de formatos tradicionales; por otro lado, la exploración de cómo las redes sociales se han convertido en un aliado indiscutible del artista como profesional en el siglo XXI, como medio sin precedentes para la difusión de su obra.

\subsection{Las redes sociales como marco creativo para los artistas}

En torno a 2008 comenzaron a aparececer propuestas artísticas muy atentas a lo que sucedía en las redes sociales y que tenían como referencia estas vías de comunicación colectivas desarrolladas en la red. Twitter, Facebook, YouTube, Blogger e Instagram han contribuido a desarrollar ingentes propuestas artísticas que ponen de manifiesto la importancia del fenómeno y la transformación del arte a través de las redes sociales. Las redes en sí y su funcionamiento psicosocial puede ser el tema central sobre el que las obras enfocan su carga significacional, pero también - sin necesidad de incluir ambos conceptos en un mismo casopueden presentarse como herramienta vertebradora del proceso y resultado de la obra materializada.

Twitter concentra una serie de condiciones que se presentan tan limitadoras como liberadoras a la hora de favorecer el ingenio y llevar a cabo proyectos creativos. El límite de 140 caracteres se convierte en "la medida" para las obras de Twitter art. Todo lo que pueda condensarse en dicha medida es válido, pudiendo quebrarse la creatividad ante tal rigidez o, por el contrario, desarrollar contenidos e 
iniciativas que adquieran significado en el cumplimiento riguroso de tal limitación informacional. En este marco de creación, conviene señalar el importante peso del artista no profesional, cuya creatividad, gracias al democratizador medio de Internet y las nuevas facilidades de la Web 2.0, puede ponerse en juego sin una adquisición pormenorizada de aptitudes técnicas. La imaginación empieza a desarrollarse a pasos agigantados en la conducta de los usuarios, permitiendo una explosión de creatividad "amateur" que inunda redes sociales como Twitter y Facebook. Este hecho hace de la expresión Twitter art un concepto amplio que abarca multitud de posibilidades en cuanto a actitudes y resultados, pero con un factor común: la intencionalidad artística (López Martín, 2012: 40).

El término Twitter art se aplica a proyectos surgidos en torno a esta plataforma de microblogging a finales de la primera década del siglo XXI. Afirma Martín Prada que uno de estos ejemplos es el proyecto titulado Auto-yo (2008) de Linda Ruiz:

\footnotetext{
Centrado en los procesos de construcción identitaria que tienen lugar en Twitter, tematiza la inducción que este servicio hace a establecer un flujo comunicativo permanente, algo que esta autora subvierte mediante un automatismo apropiacionista de fragmentos de textos encontrados en la red y, por lo tanto, no configuradores de una <<identidad >> que se pueda ir conociendo a lo largo del tiempo mediante envíos. (Martín Prada, 2012: 172)
}

En Twistori (2008), una obra de los artistas Amy Hoy y Thomas Fuchs, se recurrió a recopilar en una web todas las publicaciones de Twitter que recogían las expresiones I love, I hate, I feel, I believe, etc. Cuando el "espectador" virtual accedía a la web, podía pinchar sobre cualquiera de estas expresiones, sucediéndose con ello en la pantalla todos los tweets que las contenían. 
Imagen 1. Web del proyecto Twistori (2008)

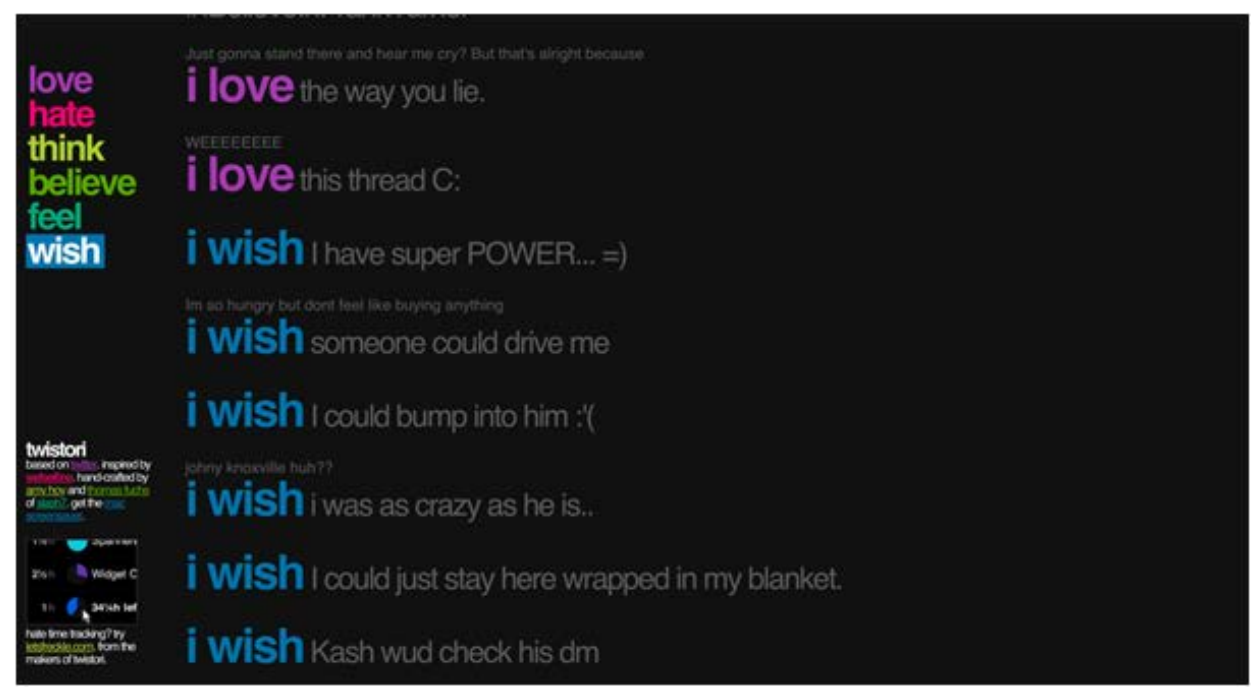

Fuente: Hoy, A. y Fuchs, T. (2008). Recuperado de http://twistori.com/\#i wish

Por su parte, la pareja belgo-holandesa conocida como JODI presentó un interesante trabajo bajo el título Twitter SK8 (2008), en el que planteaban una suerte de performance. Un conjunto de jóvenes habrían utilizado teclados de ordenador inalámbricos con ruedas, a modo de skatesboards para escribir sus mensajes, de manera que cada vez que el zapato pisase la tecla de envío, las palabras fuesen enviadas: una especie de mensajes ilegibles que cuestionaban un sistema de expresión irreflexivo, tan impulsivo como el que toma lugar en ocasiones en la propia red.

El artista Jens Wunderling, establecido actualmente en Berlín, nos sitúa en su proyecto Default to Public (2008) ante la descontextualización de los mensajes de Twitter. Wunderling extrae en tiempo real los tweets de un usuario o los de una zona geográfica y los expone de diversas formas: un timbre para la entrada de una casa en la que se muestran los tweets del propietario, una máquina que imprime tweets en tiras de papel para ser distribuidas por la ciudad, o una proyección en la superficie de un escaparate. La información difundida en Twitter se muestra, así como realmente "pública", expuesta en lugares públicos del mundo 
físico. Destacamos la performance Nothing to Tweet Home About, realizada en 2009 por An Xiao. Este artista multimedia envió por correo ordinario a una sala de exposiciones cien postales en las que escribía sus tweets acompañados de la localización geográfica desde la que habían sido publicados. Mediante esta descontextualización de los tweets, Xiao pretendía hacer reflexionar acerca de la forma de autorretratarnos diariamente en la red.

En el panorama latinoamericano queremos destacar DeepToughtV2 (2009), una instalación de la mexicana Leslie García, miembro del colectivo DreamAddictive de Tijuana. La instalación funcionaba a modo de adivina digital, que utilizaba Twitter para apropiarse de frases poéticas y premonitoras, con las que respondía a quienes le consultaban. La colaboración de los internautas consistió en enviar pensamientos profundos y elaborados, micropoemas y mensajes para ampliar la memoria de su obra. Para participar era tan solo necesario agregar el hashtag \#dtv2 en los tweets que se compartían en el proyecto (Bosco \& Caldana, 2012). Por su parte, Cory Arcangel plantea algunas series como Working on my novel (2009) o Follow my other Twitter (2011) en las que recopila patrones de repetición de los estados de los blogueros o de situaciones similares vividas por los usuarios.

En el contexto europeo, es interesante señalar cómo los artistas italianos Clemente Pestelli y Gionatan Quintini en su obra Les liens invisibles-Silence is Golden (2009) realizaron una intervención perpetua en Twitter por medio de un script que elaboraba tweets sin texto. Dicho proyecto podría ser leído como crítica a la necesidad de comunicar el estado de las acciones propias de los individuos de forma constante, planteándose como un espacio de silencio en medio del ruido informativo circundante. Haciendo uso de una estética muy similar, el colectivo Jimpunk presentó en 2012 la obra exq=.s.te =n.c\&de/s, una especie de teclado virtual (cuyas teclas eran símbolos) que permitía a cualquier internauta generar micro textos de símbolos de un máximo de 140 caracteres, y que, tras pulsar el botón $\mathrm{OK}$, enviaba los mensajes en forma de actualización de estado a la cuenta de Twitter @crashtxt. La pieza confería la posibilidad - a todos los usuarios de la red-de convertirse en coautores de una única cuenta de Twitter que dejaba de ser un lugar de publicación de contenidos personales, para transformarse en una 
cuenta plural en la que los mensajes - ilegibles - no comunicaban conceptos, sino grupos de signos sin sentido en los marcos del lenguaje convencional.

La red social Facebook también ha dejado algunos ejemplos remarcables de la utilización que hacen de ella los artistas. La red con más usuarios y seguidores en el planeta se convierte, por el momento, en un canal de enorme difusión y un altavoz de ideas y propuestas de gran alcance. Apenas cinco años después de su aparición, el artista Gordan Savicic se planteaba en su proyecto Suicide Machine (2009) facilitar al usuario una desconexión de las redes sociales, protagonizando un "suicidio" de su perfil en dichas redes, por medio de un programa que accedía a la cuenta y eliminaba automáticamente a los amigos y seguidores, para finalmente eliminar al propio usuario. En el proyecto de Savicic podemos observar un vídeo de presentación que critica la manera en que las redes sociales suprimen -en opinión del artista- las relaciones humanas con personas de nuestro entorno. La empresa del visionario Mark Zuckerberg logró finalmente que este proyecto dejase de estar en activo.

En el trabajo de Grégory Chatonsky, titulado I Would Liked to be One of You (2009), encontramos a menudo una manipulación de los flujos de información con la finalidad de crear ficciones sin fin. En este caso, se trata de una aplicación que funciona dentro de Facebook y roba los estatus de otros para publicarlos en el perfil propio. De esta manera, la información, enlaces, fotos, y reflexiones publicadas por los amigos se convierten en aportaciones del propio usuario, generando una confusión de identidad y difundiendo contenidos falsamente atribuidos al usuario.

Alessandro Ludovico y Paolo Cirio se enfrentaron también críticamente a Facebook en su obra Face-to-facebook (2011). Consiguieron extraer de la red social un millón de fotos y datos básicos de los perfiles de usuario, con los que, tras procesarlos en diversas categorías (hombre/mujer, divertido, astuto, etc.), obtuvieron unos 250.000 perfiles con los que crearon un falso sitio web de citas. 
El proyecto suscitó polémica por el uso de datos robados y la manera en que la intimidad de los usuarios era expuesta en otro contexto. Los defensores legales de Facebook les obligaron a cerrar la web de citas, pudiendo exhibir únicamente en la actualidad la documentación referente al proyecto.

Imagen 2. Falso portal web de citas desarrollado para la obra

Face-to-facebook (2011)

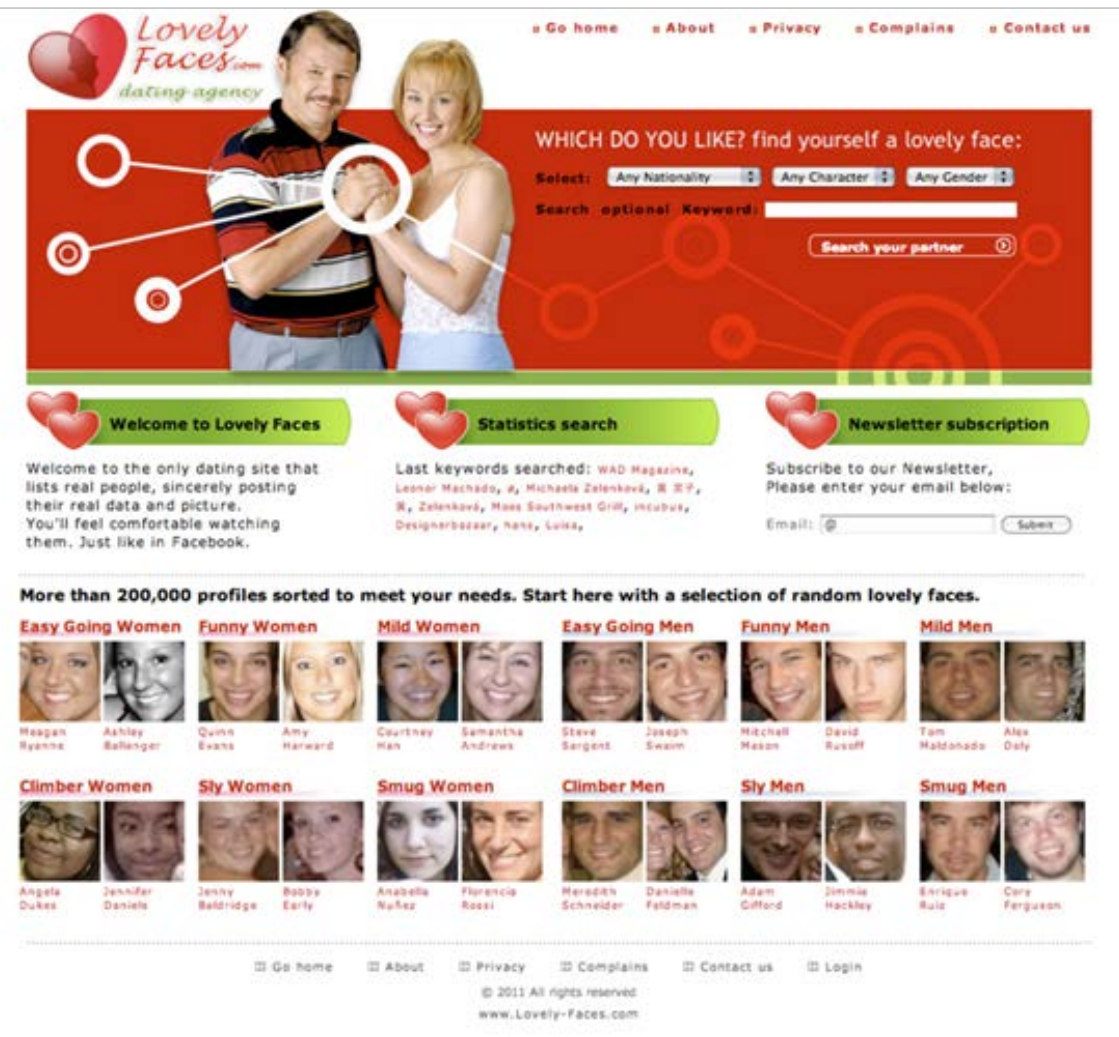

Fuente: Ludovico, P. y Cirio, P. (2011). Recuperado de https://paolocirio.net/work/face-to-facebook/

En España, el caso de Ana Riaño (Bilbao, 1985) es un ejemplo excepcional de utilización de las redes sociales como inspiración y material artístico para sus creaciones. En la serie "Redes Sociales" de 2015-2016, presenta un conjunto de 
obras en lienzo en las que reflexiona sobre la imagen que proyectamos en Internet y sobre cómo se diluye en este contexto la frontera que separa nuestra información profesional y personal. La serie se divide en dos grupos; en el primero de ellos se agrupan los perfiles de personajes en activo en el mundo del arte contemporáneo, como es el caso del crítico de arte Javier Díaz Guardiola, el artista Jeff Koons o el director del PS1 de Nueva York. En el segundo apartado encontramos una recreación de cómo la autora imagina la forma en que podrían haber sido los perfiles de conocidos artistas como Ana Mendieta y Marcel Duchamp - que desarrollaron su carrera en la era pre-Internet- Hasta un total de 50 piezas pictóricas con los perfiles de Twitter y Facebook de artistas, galeristas, directores de museos y críticos de arte toman lugar en la iniciativa. Lo singular del proyecto, quizá, es la utilización de la pintura - un medio tradicional-, para dar vida a las redes sociales de estos agentes del arte y artistas ya fallecidos que no pudieron acceder a ellas, al mismo tiempo que se realiza una reflexión de cómo serían sus hipotéticos perfiles. Su trabajo no se detiene, por tanto, en una mera captura de obras de Internet, sino que hace el ejercicio de pintar, recrear obras de otros artistas, jugar con la imaginación y con el espectador en una reflexión cargada de intención y doble sentido.

Imagen 3. Obra Ingres (2017), de Ana Riaño. Acrílico sobre papel. 110*160 cm

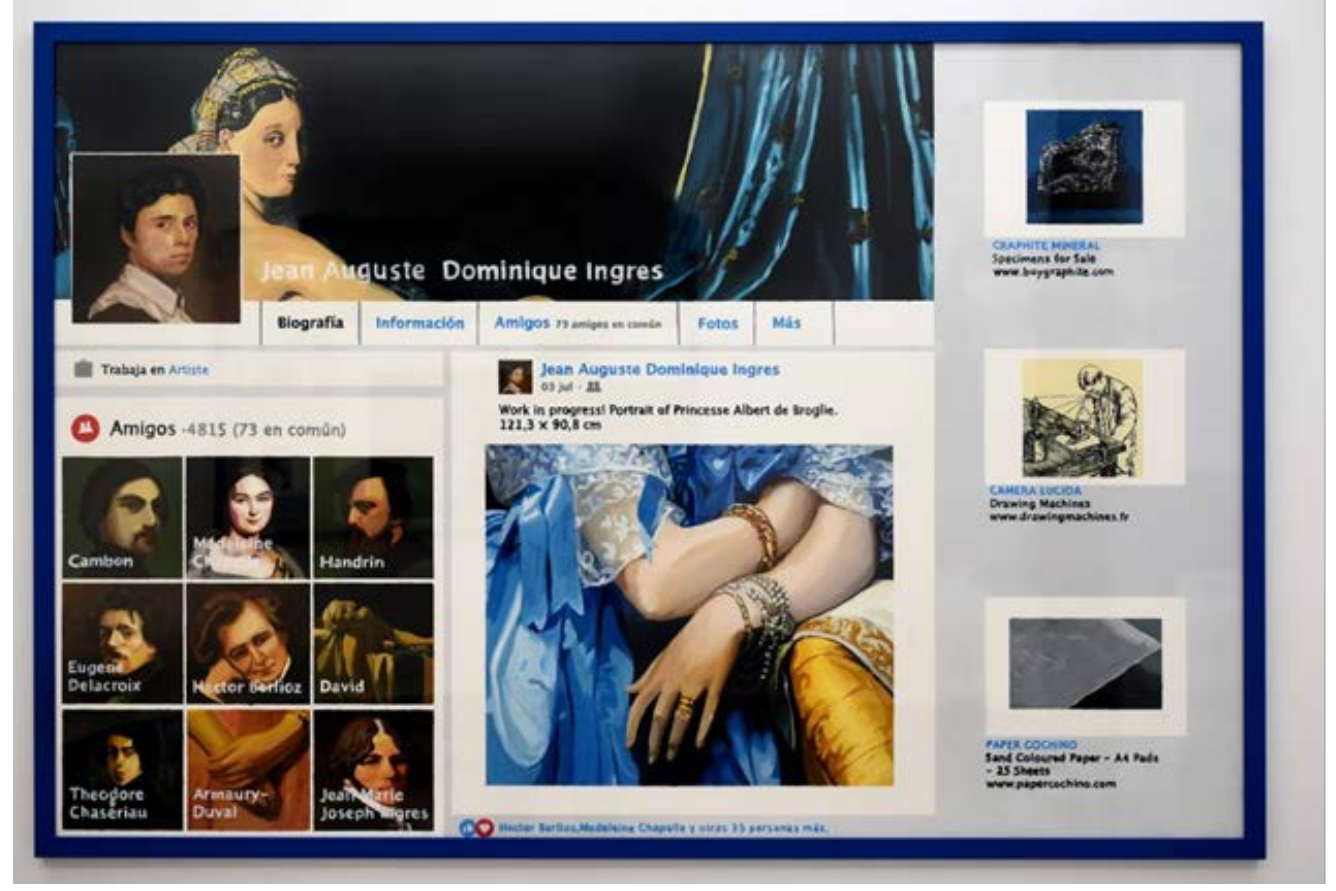

Fuente: Hornback, D. (2017). Recuperado de https://www.nuvol.com/noticies/ingres-tha-enviat-una-sollicituddamistat/ 


\subsection{Las redes sociales como canal de difusión para la obra de los artistas}

Los artistas 2.0 han entendido el poder que Internet proporciona a la hora de publicitar sus obras de arte. Plataformas como Pinterest, Tumblr, Flickr, Twitter, Facebook e Instagram se han convertido en espacios esenciales para artistas emergentes que buscan compartir sus trabajos con el mundo (pinturas, esculturas, fotografías, etc.); se trata de una forma de expandir su público y fomentar sus propias estrategias de marketing y ventas. De igual modo, artistas con carreras más consolidadas cuyas obras se exponen habitualmente en galerías y museos también se sirven de las redes sociales para promover estos eventos e invitar al espectador a conectarse con ellos, ya no solo por medio de su trabajo, sino mediante la interacción directa de ambos a través de las redes.

Esta utilización de las redes por parte de artistas independientes como plataformas de comunicación hacia la difusión de sus obras - manejadas incluso por parte de las propias instituciones artísticas- muestra una necesidad de actualización en la materia para los artistas emergentes, con el objetivo de alcanzar un uso eficiente y premeditado de las nuevas tecnologías y servicios que ayudan a difundir sus trabajos y resultados. Es en esta parcela donde subrayamos la necesidad de comprender el arte del siglo XXI como una unidad orgánica en la que intervienen diversos fenómenos de extensas complejidades al mismo tiempo. Para Jiménez (2010: 234), no estamos ante la disolución de los géneros artísticos tradicionales, pese a la "integración estética de las artes, la electrónica y la informática". El autor intuye una transformación en la figura tradicional del artista. Enlazando con la cuestión aquí tratada sobre la difusión de obras por medio de las redes sociales, encontramos también un cambio entre las relaciones entre productor y consumidor, una metamorfosis que se manifiesta en la interacción con las redes. Así, Jiménez afirma (2010:235): 
Pero se trata de un consumo público (el consumo del arte), y no privado. La relación individual entre productor (artista) y consumidor (cliente) ha desaparecido para dejar paso a una relación abstracta, configurada con las características capitalistas de la mercancía, en la que ambos experimentan su integración en canales públicos.

El reconocimiento de la importancia de las redes sociales como herramienta innovadora ha ocasionado que los artistas sean conscientes de que no solo atraen a un nuevo público, sino que se les conecta con los espectadores de su obra, promoviendo la comprensión y debate sobre su arte como nunca fue posible. El eficaz manejo de estos recursos permitirá un posicionamiento y visibilidad de los contenidos, una positiva ampliación de la red de contactos profesionales y una comunicación directa e interactiva con las audiencias interesadas en su obra. En definitiva, todo un elenco de nuevas oportunidades de crecimiento y desarrollo profesional, no exento del abandono de formatos anteriores ni del riesgo de nuevos conflictos asociados al nuevo paradigma.

\section{Conclusiones}

Una de las primeras conclusiones a las que llegamos después de consumar la presente investigación es que el uso de las redes sociales digitales en el arte se encuentra en un estado preliminar de su historia; apenas asistimos al esbozo de los primeros trazos de una relación creativa y un camino que estarán marcados por el devenir de las redes, su permanencia, o el surgimiento de otras nuevas. De todos los materiales y nuevos medios introducidos en el arte a principios del siglo XXI, este binomio conceptual redes/arte explora y a menudo subvierte los modos tradicionales de la creatividad. Los artistas que emplean estos nuevos recursos forman parte del cambio social y participan en él como actores y como creadores de un arte interactivo que se relaciona intensamente con el tiempo en el que se construye la obra. No es posible, por el momento, trazar una línea narrativa completa de la relación arte/redes, porque asistimos tan solo a sus comienzos, pero podemos vislumbrar un estrecho y proteico vínculo. 
Las redes sociales digitales han contribuido enormemente a la destrucción del concepto de "cultura de élite", impuesta en museos y galerías de arte, donde solo una minoría social parecía poder alcanzar el estatus de espectador y consumidor. Hoy en día, las revolucionarias posibilidades que nos brindan estos medios permiten una acelerada difusión informativa y la posibilidad de una intensa interacción del público con los trabajos presentados, dando paso a una nueva dimensión de participación e interpretación del arte: una "alta cultura" con "espectadores populares". Estas nuevas tecnologías virtuales y medios de participación masiva han cambiado la forma de crear, participar, comprender la cultura, democratizándola, pero también introduciendo un temor a la pérdida de intimidad y a las formas artísticas anteriores. Asimismo, han conducido al cuestionamiento sobre el futuro de las relaciones humanas, cuestiones sobre las que los artistas han reflexionado en y con estas redes.

Si Internet supuso un punto de inflexión en la producción artística aportando características propias a las obras surgidas en el contexto del Net.art, la llegada de las redes sociales digitales ha aportado un nuevo campo de reflexión y experimentación para los artistas, convirtiéndolas en soporte artístico y constructor de sus propuestas. El acercamiento entre la producción artística y las redes sociales ha generado diferentes resultados de colaboración en el binomio conceptual arte/comunicación: desde la consideración de las redes como un espacio para la divulgación de las obras, pasando por la valoración de la red social como una herramienta para realizar la obra final o como un tema repleto de significados sobre el que el artista puede reflexionar para crear y dar vida a obras basadas en presupuestos conceptuales. Uno de los retos más pronunciados que se plantean en el horizonte es el de compaginar la coherencia del relato clásico con las posibilidades técnicas, encontrando un equilibrio de recursos similar al que se adquirió en anteriores etapas de la historia del arte. Habrá que encontrar la coherencia artística con respecto a la nueva mirada de la posmodernidad, filtrada a través de los dispositivos: ¿será necesaria la gestación de nuevas estructuras discursivas verdaderamente contemporáneas y emanadas de esta nueva infraestructura? 
La influencia de la tecnología y el sistema de red en el arte han generado la última revolución artística. El carácter en ocasiones inmaterial de las obras surgidas al albor de los nuevos tiempos ha provocado una alteración en la naturaleza de la obra de arte, ha modificado el concepto tradicional de artista, ha supuesto la aparición de nuevos géneros artísticos y ha estimulado la adaptación de las instituciones artísticas y los creadores a un nuevo paradigma de comunicación y arte. 


\section{Bibliografía}

- AZAM, M. \& FEDERICO, A. (2014). Sociología del arte y análisis de redes sociales. REDES: Revista Hispana Para El Análisis De Redes Sociales, 25(2), 1-22. Recuperado de http://revistes.uab.cat/redes/article/view/v25-n2-azamdefederico/pdf-es

- BAUDRILLARD, J. (2000). Pantalla total. Barcelona: Anagrama.

- BAUDRILlARD, J. (2009). La sociedad de consumo: Sus mitos, sus estructuras. Madrid: Siglo XXI de España.

- BOSCO, R. \& CALDANA, S. (2014). Arte cinético para las redes sociales en El País. Recuperado de http://blogs.elpais.com/arte-en-la-edad-silicio/2014/05/ arte-cinetico-para-las-redes-sociales.html

- CHOZA, J. (2015). Filosofía del arte y la comunicación: Teoría del interfaz (1ạ ed.). Sevilla: Thémata.

- CLARAMONTE, J. (2016). Estética modal. Madrid: Tecnos.

- GARCÍA, N. (2012). Introducción. De la cultura postindustrial a las estrategias de los jóvenes. En GARCÍA, N., CRUCES, F. \& URTEAGA, M. (Coords.), Jóvenes, culturas urbanas y redes digitales: Prácticas emergentes en las artes, las editoriales y la música (pp. 3-24). Barcelona; Madrid: Editorial Ariel.

- GERBER, V. \& PINOCHET, C. (2012). La era de la colaboración. Mapa abreviado de nuevas estrategias artísticas. En GARCÍA, N., CRUCES, F., \& URTEAGA, M. (Coords.), Jóvenes, culturas urbanas y redes digitales: Prácticas emergentes en las artes, las editoriales y la música (pp. 45-64). Barcelona; Madrid: Editorial Ariel. 
- IZQUIERDO, V., ÁLVAREZ, P. \& NUÑO, A. (2017). Comunicación y divulgación de contenidos artísticos en las redes sociales: Facebook y Twitter. Estudios sobre el Mensaje Periodístico 23 (2), (pp. 1161-1178). Madrid: Ediciones Complutense.

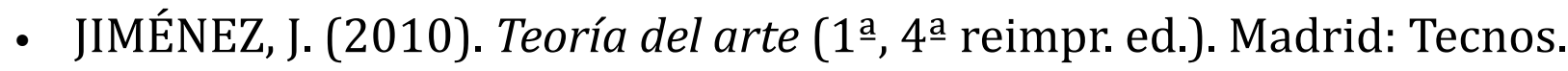

- KELLNER, D. (2011). Cultura mediática: Estudios culturales, identidad y política entre lo moderno y lo posmoderno. Tres Cantos (Madrid): Akal.

- LIPOVETSKY, G. \& SERROY, J. (2016). La estetización del mundo: Vivir en la época del capitalismo artístico (2a ed.). Barcelona: Anagrama.

- LÓPEZ MARTÍN, E. (2012). Twitter como argumento, herramienta y soporte para la producción artística contemporánea, en Forma. Revista D’Humanitats, 6, (pp.33-47). Barcelona.

- MARCHÁN FIZ, S. (2012). La estética en la cultura moderna: de la Ilustración a la crisis del Estructuralismo. Madrid: Alianza Editorial.

- MARTÍN PRADA, J. (2012). Prácticas artísticas e Internet en la época de las Redes Sociales. Madrid: Akal.

- ORTEGA, E. (2012). Aprendices, emprendedores y empresarios. En GARCÍA, N., CRUCES, F. \& URTEAGA, M. (Coords.), Jóvenes, culturas urbanas y redes digitales: Prácticas emergentes en las artes, las editoriales y la música (pp. 109130). Barcelona; Madrid: Editorial Ariel. 
- URTEAGA, M. (2012). De jóvenes contemporáneos: Trendys, emprendedores y empresarios culturales. En GARCÍA, N., CRUCES, F. \& URTEAGA, M. (Coords.), Jóvenes, culturas urbanas y redes digitales: Prácticas emergentes en las artes, las editoriales y la música (pp. 25-44). Barcelona; Madrid: Editorial Ariel. 


\section{Biografías}

Profesora Titular en Periodismo en la Facultad de Ciencias de la Información de la Universidad Complutense de Madrid. Especializada en Arte Contemporáneo ha publicado numerosos libros y artículos sobre movimientos artísticos contemporáneos. En la actualidad investiga en el campo de la Comunicación y el Arte, es miembro en el proyecto de investigación Análisis de la divulgación cultural y científica en los medios de comunicación de masas y es directora del Proyecto de Innovación Docente "Artenea: Arte y Periodismo" en la UCM. Miembro de la Asociación Madrileña y Española de Críticos de Arte, colabora como crítica en diferentes publicaciones nacionales e internacionales y programa actividades culturales y exposiciones.

\section{Violeta Izquierdo Expósito}

violeta.izquierdo@ccinf.ucm.es

http://orcid.org/0000-0002-9235-9322

Profesora Titular en Periodismo

Departamento de Periodismo y Comunicación Global

Facultad de Ciencias de la Información

Universidad Complutense de Madrid

Graduado en Periodismo por la Universidad Complutense. Es máster en Filosofía teórica y práctica por la Universidad Nacional de Educación a Distancia y, actualmente, doctorando en la Universidad Complutense, en la Facultad de Ciencias de la Información. Ha sido becario en el Departamento de Periodismo y Comunicación Global y actualmente responde como Colaborador Honorífico en el mismo. Realiza estudios superiores de Composición en el Real Conservatorio Superior de Música de Madrid. Su campo de investigación se centra en los ámbitos de la educación y creación artísticas y su connivencia con el contexto de interacción comunicacional a partir de los años 90.

\section{David Lima Guerrero}

dalima@ucm.es

http://orcid.org/0000-0001-9680-4078

Doctorando en Periodismo

Departamento de Periodismo y Comunicación Global

Universidad Complutense de Madrid 